\title{
Clinical outcome and dose volume evaluation in patients who undergo brachytherapy for angiosarcoma of the scalp and face
}

\author{
TOMOHIDE SANADA ${ }^{1}$, HIDETSUGU NAKAYAMA ${ }^{1,3}$, RYOKICHI IRISAWA ${ }^{2}$, \\ MITSURU OKUBO ${ }^{1}$, RYOJI TSUBOI ${ }^{2}$ and KOICHI TOKUUYE ${ }^{1}$ \\ Departments of ${ }^{1}$ Radiology and ${ }^{2}$ Dermatology, Tokyo Medical University Hospital, Tokyo 160-0023, Japan
}

Received August 16, 2016; Accepted November 4, 2016

DOI: $10.3892 /$ mco.2017.1155

\begin{abstract}
The present study was conducted to retrospectively evaluate survival and local control with definitive brachytherapy in patients with cutaneous angiosarcoma of the scalp and/or face, and to determine the optimal radiation dose and irradiated volume. Between November, 2009 and January, 2015, 9 consecutive patients with histologically proven angiosarcoma of the scalp and/or face who received image-guided brachytherapy were retrospectively evaluated. The median age of the patients was 83.4 years (range, 67.7-91.9 years). Of the 9 patients, 8 had no lymph node metastasis and 1 patient had cervical lymph node metastasis. The patients were irradiated with a dose of $3 \mathrm{~Gy}$ three times per week for varying lengths of time; 4 patients received a total dose of $60 \mathrm{~Gy}, 1$ received $48 \mathrm{~Gy}$ and the 4 remaining patients received $45 \mathrm{~Gy}$. The patient who received $48 \mathrm{~Gy}$ also underwent additional electron therapy of $16 \mathrm{~Gy}$ in 8 fractions. The overall survival, progression-free survival and local progression-free rates at 3 years were $50.8 \%$ [95\% confidence interval (CI): 15.6-78.1\%], 37.0\% (95\% CI: 6.8-69.3\%) and $77.8 \%$ (95\% CI: 36.5-93.9\%), respectively. The local progression-free rate in the 4 patients who received a total of $\geq 60$ Gy was statistically significantly better compared with that in the 5 patients who received a dose of $<60 \mathrm{~Gy}(\mathrm{P}=0.027)$. A total of 7 patients had grade 2 radiation dermatitis, whereas the remaining 2 patients had grade 3 dermatitis. All the patients had grade 2 alopecia. Local disease control achieved by radiotherapy resulted in higher survival. Therefore, prescribing $\geq 60 \mathrm{~Gy}$ in 20 fractions for the gross tumor volume is recommended for angiosarcoma of the scalp and face.
\end{abstract}

\section{Introduction}

Angiosarcoma is a rare aggressive malignant tumor of vascular endothelial cells accounting for $\sim 2 \%$ of soft tissue sarcomas $(1,2)$. Two-thirds of angiosarcomas occur in

Correspondence to: Dr Hidetsugu Nakayama, ${ }^{3}$ Present address: Department of Radiation Oncology, National Center for Global Health and Medicine, 1-21-1 Toyama, Shinjyuku, Tokyo 162-8655, Japan

E-mail: hnakayama@hosp.ncgm.go.jp

Key words: angiosarcoma, brachytherapy, radiotherapy cutaneous tissues (1), insidiously presenting as a bruise-like lesion or a purplish papule and exhibiting a tendency to spread hematogenously. One-third of cutaneous angiosarcomas develop in the scalp of elderly individuals (3).

The survival rate of angiosarcoma of the scalp and face has been reported to be as low as $15-45 \%$ at 5 years (4-6). It has been previously reported that a clear surgical margin is a major factor affecting survival (6). However, angiosarcoma invades extensively and is usually found beyond the surgical margin $(5,6)$. Thus, adjuvant postoperative radiotherapy has been employed to improve the outcome (4-6).

Various techniques of external beam radiotherapy have been applied for angiosarcoma of the scalp and face. However, despite the advances in external radiotherapy, due to the complex shape of the head, the dose distribution is usually inadequate. The build-up effect of high-energy photons or electrons reduces the radiation dose delivered to the scalp surface. In brachytherapy, the radiation dose is inversely proportional to the distance from the radiation source; thus, theoretically higher doses are delivered to the scalp surface and lower doses to the deep-seated area compared with external beam radiotherapy. This means that brachytherapy may be an optimal treatment modality for angiosarcoma of the scalp. Thus, Imai et al introduced brachytherapy with ${ }^{192} \mathrm{Ir}$ for angiosarcoma of the scalp, and reported that 3 patients were successfully treated using this method (7). However, there is little information on clinical outcomes and precise dose-volume evaluation of brachytherapy for angiosarcoma of the scalp. Thus, the aim of the present study was to evaluate the local control and overall survival of patients with angiosarcoma of the scalp and face who underwent brachytherapy, and determine the optimal dose volume of brachytherapy.

\section{Patients and methods}

Patients and methods. Between November, 2009 and January, 2015, 9 consecutive patients with angiosarcoma of the scalp and/or face who received image-guided brachytherapy at the Tokyo Medical University Hospital (Tokyo, Japan) were retrospectively evaluated. The characteristics of the patients are summarized in Table I. All the patients had histologically proven angiosarcoma. The median age was 83.4 years (range, 67.7-91.9 years) and the main tumor locations were as follows: Parietal area in 7 patients, temporal area in 1 and occipital area 

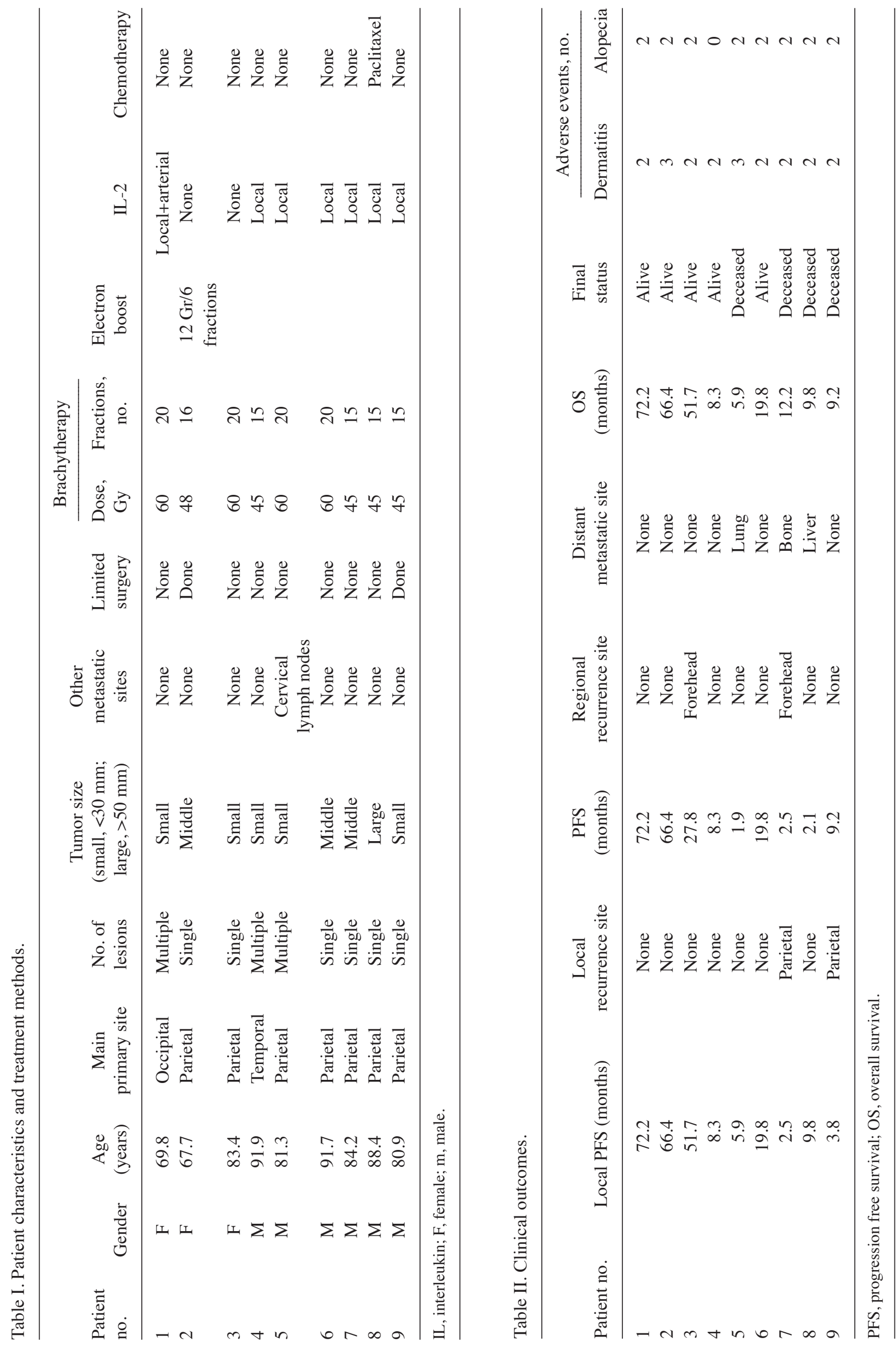

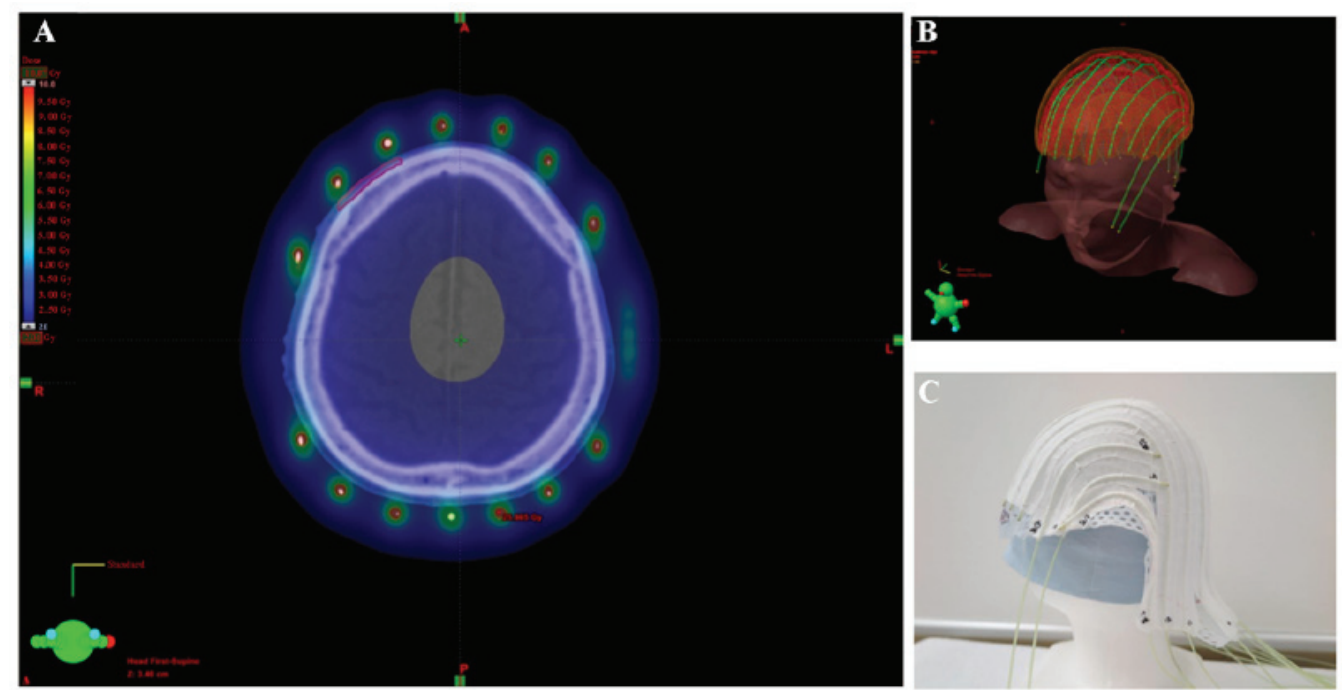

Figure 1. (A) Arrangement of radiation sources and dosimetry on computed tomography. (B) Association of radiation source arrangement with the patient's position. (C) The applicator used was individually made by pasting tubes on the mask.

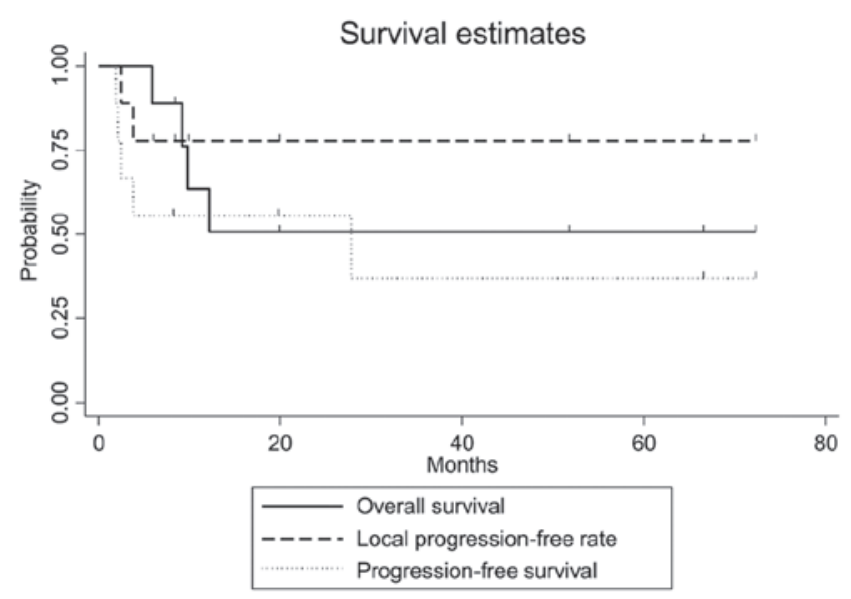

Figure 2. Kaplan-Meier's curves demonstrated that the overall survival, progression-free survival and local progression-free rate at 3 years were $50.8 \%$ [95\% confidence interval (CI): $15.6-78.1 \%], 37.0 \%$ (95\% CI: 6.8-69.3\%) and $77.8 \%$ (95\% CI: 36.5-93.9\%), respectively. The overall survival, progression-free survival and local progression-free rates at 1 year were $63.5 \%$ (95\% CI: $23.8-86.7 \%$ ), $55.6 \%$ (95\% CI: $20.4-80.5 \%$ ) and $77.8 \%$ (95\% CI: 36.5-93.9\%), respectively.

in 1 patient; 3 patients had tumors in multiple sites, and 6 had tumors in a single location on the scalp area. Of the 9 patients, 8 had tumors in the scalp area and 1 had a tumor in the facial area; 1 patient also had metastasis to the cervical lymph nodes.

The patients were immobilized with a custom-made thermoplastic mask. Applicators were fixed to avoid displacement of the mask using cotton fabric tapes at intervals of $2 \mathrm{~cm}$ on the outer side (Fig. 1), except for the applicators of the first patient, which were fixed on the inner side of the mask.

A visible lesion of angiosarcoma was defined as the gross tumor volume (GTV); the clinical target volume (CTV) was determined as the GTV plus an adequate margin along the skin and a 1-2-mm margin perpendicular to its axis. The planning target volume (PTV) was defined as the CTV with a $10-\mathrm{mm}$ margin along the skin and a 1-2-mm margin perpendicular to its axis. The skull, cerebrum and cerebellum were

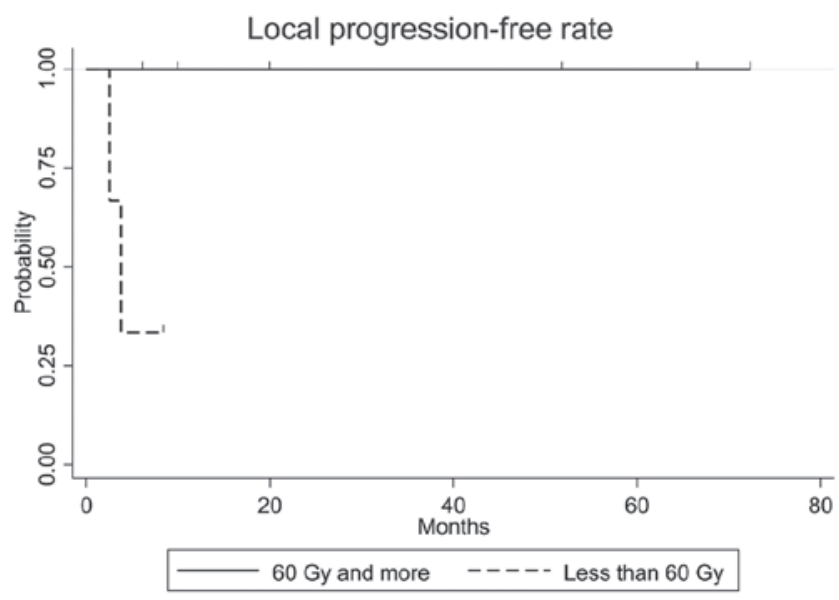

Figure 3. The local progression-free rate in patients who received a dose of $\geq 60$ Gy was found to be statistically significantly higher compared with that in patients who received a dose of $<60 \mathrm{~Gy}(\mathrm{P}=0.027)$.

also delineated. All the patients underwent image-guided brachytherapy based on computed tomography (CT) images. The arrangement of radiation sources of ${ }^{192} \mathrm{Ir}$ was adequately placed to cover the entire GTV within the line of $3 \mathrm{~Gy}$, and PTV within the line of 2 Gy. Heterogeneity of dose distribution to the scalp was geometrically optimized using a brachytherapy 3-dimensional planning system (BrachyVision; Varian Medical Systems Inc., Palo Alto, CA, USA). Radiation sources of ${ }^{192} \mathrm{Ir}$ were placed through the applicator using the VariSource afterloading system (Varian Medical Systems).

The patients were irradiated with a dose of 3 Gy three times per week for varying lengths of time. A total of 4 patients received a total dose of $60 \mathrm{~Gy}, 1$ received 48 Gy and 4 patients received $45 \mathrm{~Gy}$. The patient who received $48 \mathrm{~Gy}$ also underwent additional electron therapy of $16 \mathrm{~Gy}$ in 8 fractions. The equivalent dose delivered at 2.0 Gy per fraction was calculated by the linear-quadratic model with $\alpha / \beta$ ratios of 10 (8). The cervical lymph node metastasis of the patient was additionally treated by external radiotherapy at a dose of $50 \mathrm{~Gy}$ in 25 fractions. 
Two patients underwent limited surgery prior to radiotherapy and 7 patients underwent recombinant interleukin-2 (rIL-2) immunotherapy; of those 7 patients, 6 received a local injection and 1 was treated with both local and arterial injections. One patient received sequential chemotherapy with paclitaxel following completion of radiotherapy: Paclitaxel at a dose of $100 \mathrm{mg} / \mathrm{m}^{2}$ was administered once per week; however, after two administrations, paclitaxel was discontinued due to cholangitis.

Follow-up was principally performed by dermatologists every 3 months. The adverse events were assessed according to the Common Terminology Criteria for Adverse Events, version 4.0 (https://evs.nci.nih.gov/ftp1/CTCAE/CTCAE _4.03_2010-06-14_QuickReference_5x7.pdf).

Dose evaluation. Doses of 100, 95, 90, 85, 80, 70, 60, 50, 10 and $5 \%$ of the PTV and the maximal doses were evaluated in the skull, cerebrum and cerebellum using the BrachyVision brachytherapy 3-dimensional planning system (Varian Medical Systems Inc.). The data were used for necessary dose analysis.

Statistical analysis. The overall survival time was calculated from the first day of brachytherapy to the date of death from any cause. Hospital visits provided data on surviving patients. The progression-free survival time was calculated from the first day of brachytherapy to the date of the first relapse at any site or the date of death. The local progression-free rate was calculated according to to the date of relapse in irradiated sites. Survival time was calculated using the Kaplan-Meier method and the difference was compared using the log-rank test. Paired variables were compared by the Wilcoxon matched-paired signed-rank test. Stata statistical software, version 13 (StataCorp., College Station, TX USA) was used. A probability value of $<0.05$ was considered to be statistically significant.

\section{Results}

The clinical outcomes are presented in Table II. The median observation period was 12.2 months (range, 5.9-72.2 months). The overall survival, progression-free survival and local progression-free rates at 3 years were $50.8 \%$ [95\% confidence interval (CI): $15.6-78.1 \%$ ], $37.0 \%$ (95\% CI: 6.8-69.3\%), and $77.8 \%$ (95\% CI: 36.5-93.9\%), respectively (Fig. 2). The equivalent fractionated and total doses according to the biological effectiveness for GTV and PTV are shown in Table III. Of the 9 patients, 2 developed local infield recurrence within 4 months following completion of brachytherapy with a radiation dose of 45 Gy in 15 fractions. Three patients who were treated with a dose of $\geq 60$ Gy had no local recurrence and survived without late complications. The patient with the cervical lymph node metastasis who underwent radiotherapy of $\geq 60$ Gy developed lung metastasis 2 months after the completion of radiotherapy. The local progression-free rate in patients who received a dose of $\geq 60$ Gy was statistically significantly higher compared with that in patients who received a dose of $<60 \mathrm{~Gy}(\mathrm{P}=0.027$, Fig. 3). Of the 2 patients who experienced recurrence in the forehead outside the irradiated area, 1 was successfully treated by local injection of rIL-2, but the other patient developed bone
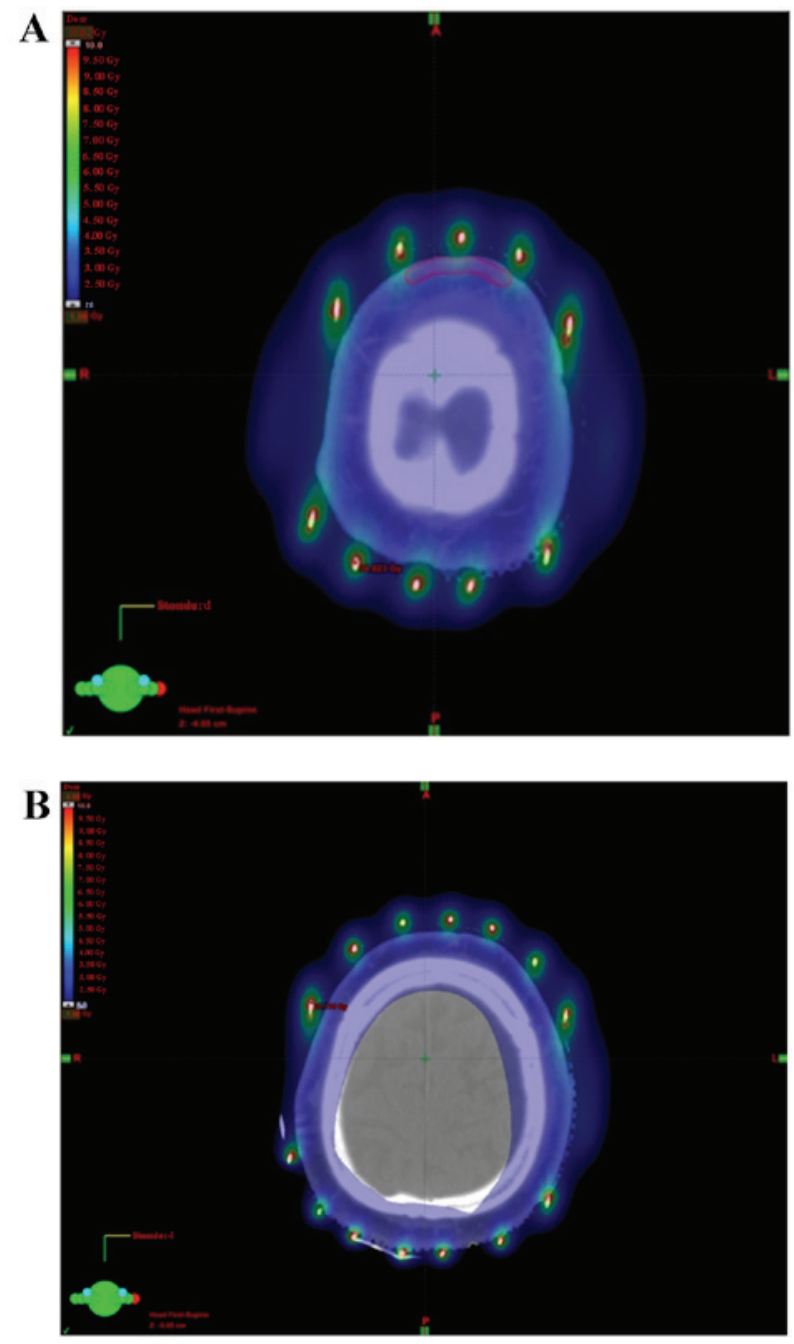

Figure 4. Dose distribution in a 88-year-old man with angiosarcoma of the parietal region. (A) Dosimetry showed the planning target volume (PTV) was covered by $>3$ Gy and (B) the radiation dose to the cerebrum was reduced.

metastasis. Distant metastases were observed in the bone, lung, or liver, and the patient with lung metastasis had developed cervical lymph node metastasis prior to brachytherapy. The dose-volume for organs at risk is shown in Table IV. The maximum doses to the skull, cerebrum and cerebellum were statistically significantly lower compared with GTV $(\mathrm{P}<0.01)$.

As regards adverse events, all the patients had grade 2 alopecia; 7 and 2 patients had grade 2 and 3 radiation dermatitis, respectively; none experienced grade $\geq 4$ adverse events.

\section{Discussion}

Angiosarcoma arising from endothelial cells is known to have a propensity to spread hematologenously. A report of 95 autopsies in Japanese patients demonstrated that the most common sites of metastasis were the lung, bone and liver (9). In this study, 3 patients experienced hematologenous metastasis. By contrast, 4 of the 9 patients treated by brachytherapy exhibited prolonged survival without local or distant metastasis at a median of 38.4 months. Guadagnolo et al reported that approximately two-thirds of the patients experienced local recurrence in an analysis of 70 patients with angiosarcoma, and 

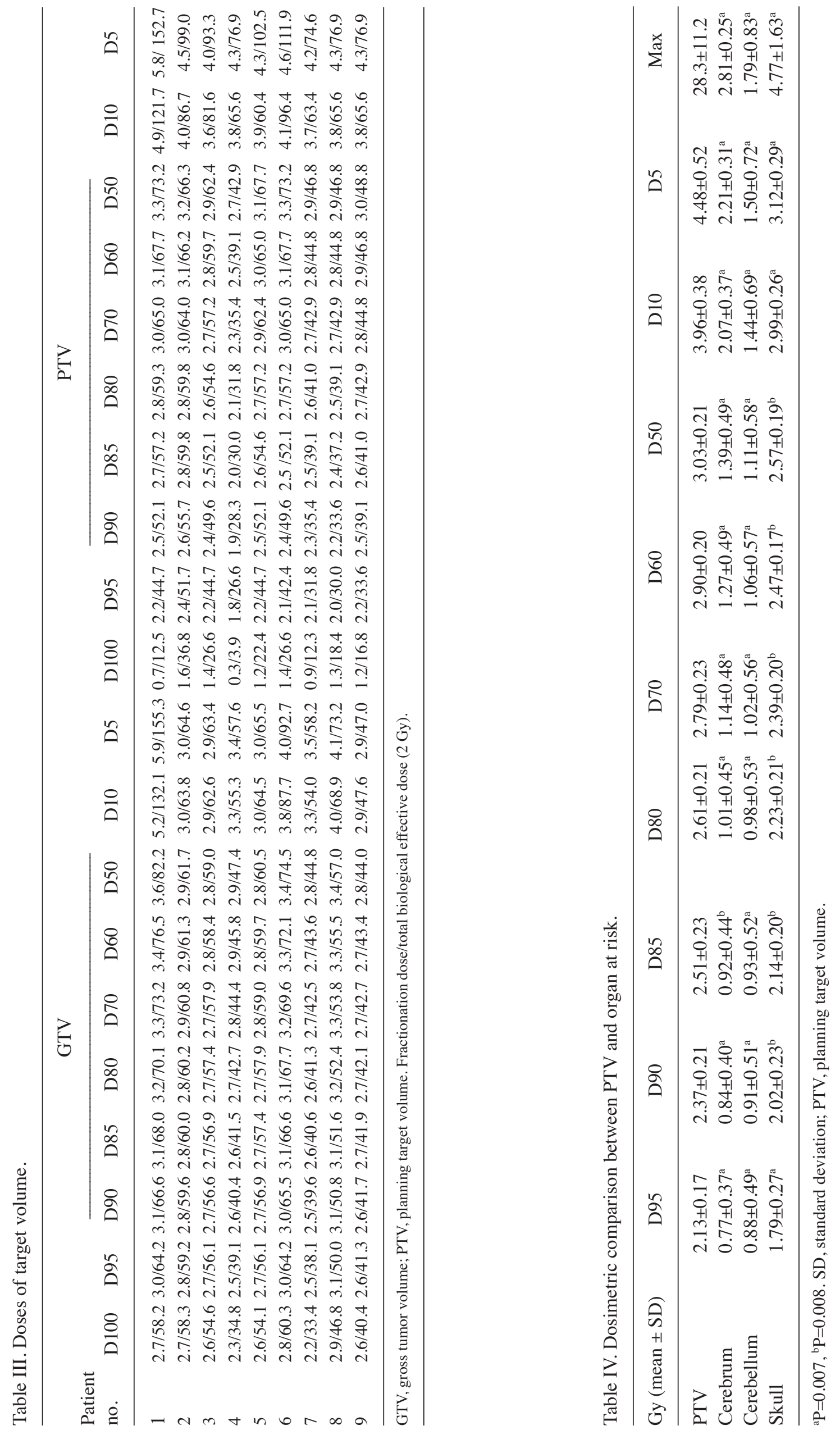
survival time was short following relapse. The authors denoted that local recurrence was associated with a high mortality rate and that local disease control was crucial (4).

Reports on angiosarcoma of the scalp and face included a variety of treatments. Several reports demonstrated that the addition of radiotherapy following surgery was the optimal approach to achieving local control and improving survival (4-6). Patel et al demonstrated that surgery followed by radiotherapy improved local control in a series of 55 patients (5). Pawlik et al also reported that the addition of radiotherapy was a prognostic factor for overall survival, along with age and $\mathrm{T}$ stage in an analysis of 29 patients (6); they demonstrated that radiotherapy significantly decreased the mortality risk (hazard ratio $=0.16$; $\mathrm{P}<0.01$ ) using a multivariate analysis.

As regards other prognostic factors, Abraham et al reported that surgical margin negativity was a significant prognostic factor (3), whereas others were not (4-6). Pawlik et al employed radiotherapy, although no tumors were detected beyond the surgical margin (6). As for reports from the United States, surgery was the first option and radiotherapy was secondary; whereas in a Japanese study, 42-100\% of the patients underwent radiotherapy alone (10-14), in the United States study only 7-39\% underwent radiotherapy alone (3-6). Regarding the local control rates, 44-67\% was reported by Japanese studies $(10,12-14)$ and it was comparable to $47-52 \%$ reported by studies from the United States $(5,6,12)$. These results suggested that surgery may not be required for angiosarcoma of the face and scalp.

In the present study, a dose of $\geq 60$ Gy achieved significantly better local control compared with a dose of $<60$ Gy. Of the 9 patients who underwent brachytherapy of $\geq 60$ Gy at the $50 \%$ dose (D50\%), 3 survived. Hata et al also reported results with definitive external radiotherapy for 17 patients (10). All the tumors regressed with radiotherapy; however, 2 patients had a local relapse and 5 relapsed at other sites of the scalp. The authors reported that a dose of 50-60 Gy at 2 Gy per fraction attained local control when the tumor did not invade deeper tissues. Ogawa et al reported clinical results from 14 patients treated with definitive radiotherapy: The 10 patients who received a dose of $\geq 70$ Gy did not develop local relapse, but 4 patients who received a dose of $<70$ Gy relapsed. Of the 11 patients treated with surgery followed by radiotherapy, none relapsed with a dose of $\geq 60 \mathrm{~Gy}$, but 2 relapsed when a dose of $<60$ Gy was administered (12). Based on those results and ours combined, a brachytherapy dose of 60 Gy may be adequate to obtain good local control for angiosarcoma.

Various external radiotherapy techniques have applied homogenous irradiation to the surface of the scalp. Able et al reported a technique of six-field electron therapy to the entire scalp (15). Akazawa et al introduced a parallel-opposed lateral field method using both photons and electrons (16), which was verified by Tung et al (17). Kinard et al reported a technique using four-arc photons (18). Those reports were technical, or included only a paucity of clinical data. Ostheimer et al described the doses to the target and organs at risk in clinical cases for intensity-modulated radiotherapy for the scalp (19); they reported that the mean and maximum brain doses ranged from 43 to $51 \%$ and from 101 to $109 \%$, respectively, compared with the prescribed dose. These doses were higher compared with those in our methods. In external photon therapy, an adequate bolus may be required to increase the dose to the skin surface due to the build-up phenomenon. In our image-guided brachytherapy method, an adequate dose to the scalp was feasible and the dose to the cerebrum was significantly reduced (Fig. 4).

In this study, 1 patient with cervical lymph node metastasis developed distant metastasis. Other reports also demonstrated that patients with cervical lymph node metastasis are prone to develop distant metastases $(10,13)$. Paclitaxel and doxorubicin have been used in such patients with a curative intent (20). Sasaki et al reported that the combination of rIL-2 and radiotherapy led to prolonged survival (14). In our study, recurrent lesions in the forehead were successfully treated by local rIL-2 injections. The evidence base for the regimen of chemotherapy is limited (2); however, adding chemotherapy to radiotherapy may improve local control and survival. The small number of patients in the present study did not allow for clarification of prognostic factors, such as combined chemotherapy, age and tumor size.

A frequent scalp relapse outside the irradiated fields was reported, but a precise recurrence site has not been described (5,10-12). Our study demonstrated that 2 of the 9 patients relapsed on the cutaneous area of the forehead, suggesting that the entire scalp and forehead should be included in the irradiated volume.

In conclusion, brachytherapy of $60 \mathrm{~Gy}$ at the GTV covering PTV within the 40-Gy line was an effective and less toxic treatment method for angiosarcoma.

\section{References}

1. Penel N, Marréaud S, Robin YM and Hohenberger P: Angiosarcoma: State of the art and perspectives. Crit Rev Oncol Hematol 80: 257-263, 2011.

2. Young RJ, Brown NJ, Reed MW, Hughes D and Woll PJ: Angiosarcoma. Lancet Oncol 11: 983-991, 2010.

3. Abraham JA, Hornicek FJ, Kaufman AM, Harmon DC, Springfield DS, Raskin KA, Mankin HJ, Kirsch DG, Rosenberg AE, Nielsen GP, et al: Treatment and outcome of 82 patients with angiosarcoma. Ann Surg Oncol 14: 1953-1967, 2007.

4. Guadagnolo BA, Zagars GK, Araujo D, Ravi V, Shellenberger TD and Sturgis EM: Outcomes after definitive treatment for cutaneous angiosarcoma of the face and scalp. Head Neck 33: 661-667, 2011.

5. Patel SH, Hayden RE, Hinni ML, Wong WW, Foote RL, Milani S, Wu Q, Ko SJ and Halyard MY: Angiosarcoma of the scalp and face: The Mayo Clinic experience. JAMA Otolaryngol Head Neck Surg 141: 335-340, 2015.

6. Pawlik TM, Paulino AF, McGinn CJ, Baker LH, Cohen DS, Morris JS, Rees R and Sondak VK: Cutaneous angiosarcoma of the scalp: A multidisciplinary approach. Cancer 98: 1716-1726, 2003.

7. Imai M, Nishimura T, Nozue M, Suzuki K, Kaneko M and Nimi M: The ${ }^{192} \mathrm{Ir}$ surface-mold technique for a whole scalp irradiation. J Jpn Soc Thre Radiol Oncol 11: 27-31, 1999.

8. Fowler JF: The linear-quadratic formula and progress in fractionated radiotherapy. Br J Radiol 62: 679-694, 1989.

9. Kitagawa M, Tanaka I, Takemura T, Matsubara $\mathrm{O}$ and Kasuga T: Angiosarcoma of the scalp: Report of two cases with fatal pulmonary complications and a review of Japanese autopsy registry data. Virchows Arch A Pathol Anat Histopathol 412: 83-87, 1987.

10. Hata M, Wada H, Ogino I, Omura M, Koike I, Tayama Y, Odagiri K, Kasuya T and Inoue T: Radiation therapy for angiosarcoma of the scalp: Treatment outcomes of total scalp irradiation with X-rays and electrons. Strahlenther Onkol 190: 899-904, 2014

11. Miki Y, Tada T, Kamo R, Hosono MN, Tamiya H, Shimatani Y, Tsutsumi S, Ogino R and Miki Y: Single institutional experience of the treatment of angiosarcoma of the face and scalp. Br J Radiol 86: 20130439, 2013. 
12. Ogawa K, Takahashi K, Asato Y, Yamamoto Y, Taira K, Matori S, Iraha S, Yagi N, Yogi A and Haranaga S: Treatment and prognosis of angiosarcoma of the scalp and face: A retrospective analysis of 48 patients. Br J Radiol 85: e1127-e1133, 2012.

13. Ohguri $\mathrm{T}$, Imada $\mathrm{H}$, Nomoto $\mathrm{S}$, Yahara $\mathrm{K}$, Hisaoka $\mathrm{M}$, Hashimoto H, Tokura Y, Nakamura K, Shioyama Y and Honda H: Angiosarcoma of the scalp treated with curative radiotherapy plus recombinant interleukin-2 immunotherapy. Int J Radiat Oncol Biol Phys 61: 1446-1453, 2005.

14. Sasaki R, Soejima T, Kishi K, Imajo Y, Hirota S, Kamikonya N, Murakami M, Kawabe T, Ejima Y, Matsumoto A and Sugimura K: Angiosarcoma treated with radiotherapy: Impact of tumor type and size on outcome. Int J Radiat Oncol Biol Phys 52: 1032-1040, 2002.

15. Able CM, Mills MD, McNeese MD and Hogstrom KR: Evaluation of a total scalp electron irradiation technique. Int J Radiat Oncol Biol Phys 21: 1063-1072, 1991.

16. Akazawa C: Treatment of the scalp using photon and electron beams. Med Dosim 14: 129-131, 1989.
17. Tung SS, Shiu AS, Starkschall G, Morrison WH and Hogstrom KR: Dosimetric evaluation of total scalp irradiation using a lateral electron-photon technique. Int J Radiat Oncol Biol Phys 27: 153-160, 1993.

18. Kinard JD, Zwicker RD, Schmidt-Ullrich RK, Kaufman N and Pieters R: Short communication: Total craniofacial photon shell technique for radiotherapy of extensive angiosarcomas of the head. Br J Radiol 69: 351-355, 1996.

19. Ostheimer C, Janich M, Hübsch P, Gerlach R and Vordermark D: The treatment of extensive scalp lesions using coplanar and non-coplanar photon IMRT: A single institution experience. Radiat Oncol 9: 82, 2014.

20. Italiano A, Cioffi A, Penel N, Levra MG, Delcambre C, Kalbacher E, Chevreau C, Bertucci F, Isambert N, Blay JY, et al: Comparison of doxorubicin and weekly paclitaxel efficacy in metastatic angiosarcomas. Cancer 118: 3330-3336, 2012. 